\title{
Incentive Based Multi-Objective Optimization in Electric Vehicle Navigation including Battery Charging
}

\author{
Tomohisa Hayakawa * Kyohei Ishikawa* Jun-ichi Imura * \\ Hideaki Tanaka ${ }^{* *}$ Masumi Toyoshima ${ }^{* *}$ Akihito Iwai ${ }^{* * *}$ \\ * Tokyo Institute of Technology, Tokyo, Japan \\ (e-mail: \{hayakawa,ishikawa,imura\}@cyb.mei.titech.ac.jp) \\ ** DENSO CORPORATION, Aichi, Japan \\ (e-mail: \\ \{HIDEAKI_T_TANAKA,MASUMI_TOYOSHIMA\}@denso.co.jp) \\ *** DENSO International America, Inc., Southfield, USA \\ (e-mail: AKIHITO_IWAI@denso-diam.com)
}

\begin{abstract}
This paper proposes a framework for a navigation system of electric vehicles (EVs) that minimizes the expectation of the energy consumption of the entire users while enhancing the Quality of Life (QoL), i.e., reducing the travel time and cost in our context, of individual users. To this end, we provide users optional flexibility of selecting a preferable route based on the individual travel time and cost among the multiple candidates indicated by the system, while use an incentive approach to make users select a route that requires as low energy consumption as possible. We show by numerical simulations on Chukyo Area in Japan that the proposed method is effective.
\end{abstract}

Keywords: Electric vehicles, Incentive, Smart charge, Navigation, Quality of Life, Dijkstra algorithm

\section{INTRODUCTION}

Motivated by recent remarkable advancement of information technology such as cloud computing and of rapid penetration of new infrastructure such as renewable energy and EVs, much attention has been most recently attracted on the developments of new generation energy management systems, which manage energy of the entire society effectively in a gross while raising its quality of life (QoL). The paper focuses on a navigation system of EVs and aims at developing the system that appropriately optimizes the profit of the entire society such as the minimization of its electricity consumption, in addition to the profit of the units such as individual travel time and cost.

In the energy basic plan of Japan, it is assumed that the ratio of next-generation cars mainly on EVs among unit sales of new cars is increased by approximately $50 \%$ by 2020 . In addition, the number of battery chargers is also planned to be increased to 2 million from 20,000 for the standard-charging type, and to 5,000 from 160 for the quick-charging type [Ministry of Environment, 2010, GoGoEV, 2013]. However, the mileage of EVs is still shorter than that of a gasoline car, and its charging time at the charging place is consequently longer. As an example, the mileage in one charge is around $180 \mathrm{~km}$ to $220 \mathrm{~km}$ even for the latest $\mathrm{EV}$, and is further shortened by the use of highways and air conditioners. In addition, we need approximately 30 minutes for full charge even in the case of quick-charging. Thus, for the long-distance run of EVs to need battery charging on the way, the optimal route planning methods including the charging plan such as an optimal charging place and an minimum charge quantity have been intensively studied [Kato, 2010, Artmeier, Haselmayr, and Sachenbacher, 2010, Eisner, Funke, and Storandt, 2011, Siddiqi, Shiraichi, and Sit, 2011, Storandt, 2012]. These methods provide a route that is optimal from the individual user point of view. Thus when many individual users utilize the route calculated in such a way, the traffic jam and/or the congestion at the charging place may occur from the global point of view. On the other hand, if the route is calculated from the viewpoint of the environmental aspects of the entire society, the profit of the individual users may be ignored. Hence, it is indispensable to build a platform where benefits for individual users and the entire society are nicely balanced in an appropriate way.

In this paper, we propose a multi-objective optimization framework of navigation systems for EVs minimizing the expectation of the total electricity consumption of the users while enhancing the QoL of users by giving flexibility of selecting a preferable route based on the individual travel time and cost among the multiple candidate routes suggested by the system. Specifically, the proposed system is assumed to be built for a user to choose a route with as small electricity consumption as possible by controlling the travel cost, which is one of the QoL parameters, using incentive. It is expected that this system can reduce the electricity consumption of EVs of the entire users without decreasing the QoL of individual users. The system is supposed to be implemented on the cloud-type central com- 
puting system, which provides high-speed communication among the system and cars, aggregates global information, and performs efficient optimal computation. We show that the proposed system is effective by conducting numerical simulations in the Chyukyo (greater Nagoya) area in Japan with traffic information for each section and information on the battery charging points currently set up on the road network.

Notable results related to this paper are given in Kanazawa et al. [2009, 2013]. Specifically, Kanazawa et al. [2009] introduces subsidy and capitation tax, which correspond to positive and negative incentive, in the context of replicator dynamics and investigates how the incentive can be used to bring the population state to a desirable state. This idea is further extended in Kanazawa et al. [2013] to characterize minimum latency flow in the Braess graph using the game theory. Our work, however, is distinct from those results in that Kanazawa et al. [2009, 2013] focus on stability analysis of the unique equilibrium flow and do not discuss the flexibility of users' choice of routes.

\section{PROPOSED FRAMEWORK}

\subsection{Change of Tendency in Selecting a Route by Incentive}

When the current position and the destination are given, it is natural that the drivers are likely to choose a route which is less time-consuming and less expensive. Consider the situation where there are $m$ relevant routes to the destination. In this paper we assume that an important property of these routes is characterized by energy (electricity) consumption $(E)$, travel time $(T)$, and monetary cost, or simply "cost" in short, $(C)$ so that we denote these $m$ routes by $\operatorname{Route}_{i}\left(E_{i}, T_{i}, C_{i}\right), i=1, \ldots, m$. (We also interchangeably use Route $i$ to denote $\operatorname{Route}_{i}\left(E_{i}, T_{i}, C_{i}\right)$ in this paper.)

These routes can be plotted on the $T$ - $C$ plane with the amount of $E$ represented by a circle of corresponding size. Figure 1 shows a representative example where there are three possible routes plotted. The lower left region (densely colored region) is more preferable for the drivers. Route 1 is the most time effective but most costly and Route 3 holds the opposite. Route 2 is plotted in the most densely colored region among the three routes and it should be the most chosen route by the drivers. For the three routes, our aim is to make Route 2, which is the most energy efficient, more attractive by giving incentive so that more drivers take Route 2. Note that giving positive incentive to a certain route corresponds to the fact the circle plotted on the $T-C$ plane is shifted downward so that the circle comes into the denser area.

When there are several possible routes to take, however, the drivers may choose different routes. These routes may differ individually or may be different depending on the situation that the driver is facing to. For example, in the morning the drivers may want to rush to their workplace so that the cost may be less important than the travel time to the destination. On the other hand, on holidays the drivers may choose rather less costly routes for their destinations. In general, the decision is made based on the tradeoff between total travel time that they take and the cost for electricity consumption and highway toll.

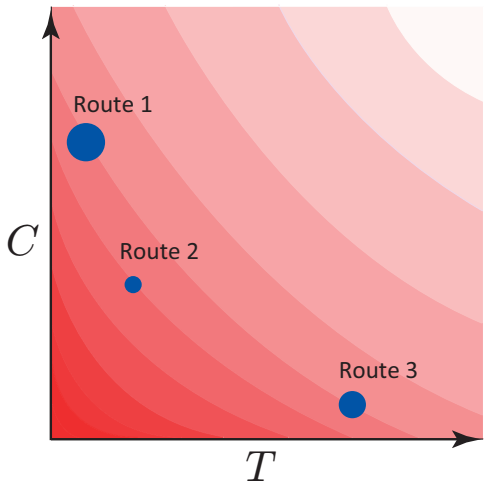

Fig. 1. $C-T$ diagram. The lower left region (densely colored region) is more preferable for the drivers.

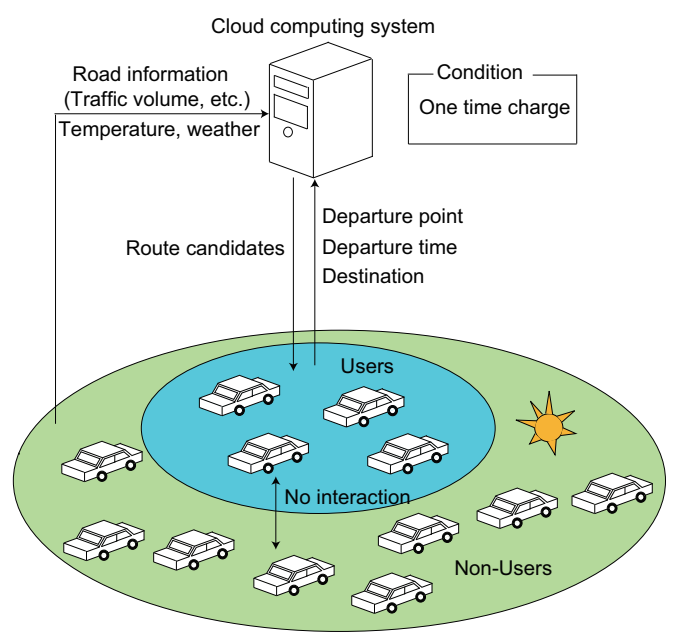

Fig. 2. Grand scheme of the proposed framework.

\subsection{Grand Scheme and Objectives}

To present more specific problem formulation, we make the following assumptions in this paper.

Controller Platform In Fig. 2, the proposed grand scheme is described. Specifically, a cloud-type computing system is assumed to be available for calculating the incentive. In the future car navigation service platform, the use of the cloud systems is assumed to be standard thereby all the information necessary for calculating a right incentive can be collected in a central way.

Controlled Plant (EVs) and the Surrounding Environments In this study we assume that only relatively low percentage of the entire vehicles have installed this route navigation system such that change of tendency of route selection by user $j$ of this system does not affect the choice of routes $\operatorname{Route}_{i}\left(E_{i}, T_{i}, C_{i}\right), i \neq j$. This assumption makes the incentive calculation extremely simple.

Calculation of Candidate Routes The cloud system presents to each user several routes with different characteristics. Specifically, for the entire benefit, it is desirable to designate all the users to take the most energy efficient route. As explained in the Introduction, however, the nonselective way of offering the route does not maintain the user's QoL. Hence, in the proposed framework we relax 
the above problem and, instead, consider the optimization problem

$$
\min \mathbb{E}\left[E_{k}\right],
$$

where $\mathbb{E}[\cdot]$ denotes the expectation, so that we attempt to minimize the total energy consumption in a statistical sense. Specifically, the idea of optimizing the expectation is predicated on the presumption that both the interpersonal and the intrapersonal preference difference in route selection should be evaluated as a long-run average for the incentive calculation as discussed in the following section.

\section{INCENTIVE-BASED MULTI-OBJECTIVE OPTIMIZATION}

In order to develop the above-mentioned framework, in this section we formulate the way of deriving the relevant route candidates and its corresponding appropriate incentive.

\subsection{General Framework and Flow of Incentive Calculation}

To begin with formulating the general framework, we list the flow of calculating appropriate incentive for having better prospect as shown below:

(1) The user designates the departure point, the departure time, and the destination.

(2) The cloud system calculates $\left(T_{i}, C_{i}, E_{i}\right)$ for Route $i$, $i=1, \ldots, m$.

(3) The cloud system calculates incentive for Route $i$, $i=1, \ldots, m$.

(4) Eliminate irrelevant routes from the route candidates. (See Sections 3.3 and 3.5 for details.)

(5) Repeat the procedures (3) and (4) until the relevant number of route candidates are determined.

\subsection{Estimation of Travel Time and Cost for Candidate Routes}

Using the Dijkstra algorithm (considering the case where the vehicle has to stop by a charge station), the cloud computing system calculates the estimated travel time, cost, and energy consumed for the set of possible routes to the destination. This result can be plotted on the $T-C$ plane as in Fig. 1.

Note that the set of routes may include the routes which is both time consuming and costly and hence are not relevant. Furthermore, there may be two or more routes which are almost the same such that one of the routes should be left selective for the users and the others should be eliminated from the candidates. The procedure of selecting relevant routes is presented in the following sections.

\subsection{Selection of Route Candidates by Clustering}

Step 1. Consider the most energy efficient route on the $T-C$ plane. If some of the routes are very close to the most energy efficient route, then they are identified as the most energy efficient route and eliminated from the $T-C$ plane. We call this the clustering procedure. For example, if Route $i$ and Route $j$ are plotted close to each other and if $E_{i}<E_{j}$, then Route $j$ is identified as Route $i$ so that if

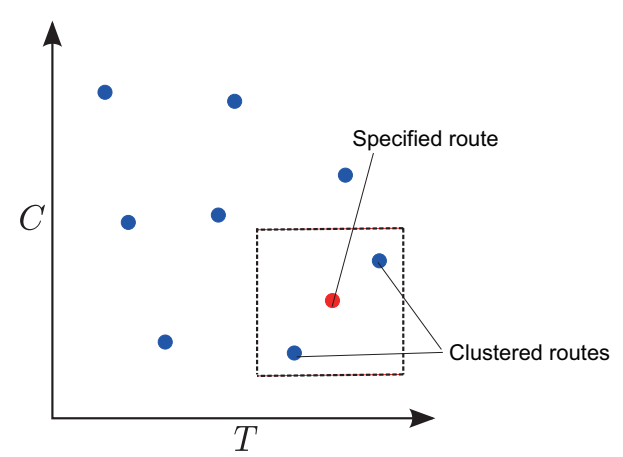

Fig. 3. Relationship between the selected path and the eliminated paths in the clustering procedure

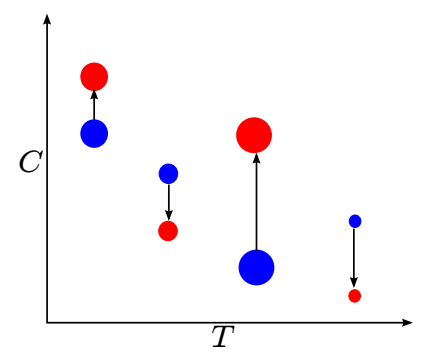

Fig. 4. Typical tendency of route properties shifted by the incentive. Energy-efficient routes indicated by smaller circles shift downward and vice versa.

$$
\begin{aligned}
\left|T_{i}-T_{j}\right| & \leq T D_{i}, \\
\left|C_{i}-C_{j}\right| & \leq C D_{i},
\end{aligned}
$$

where $T D_{i}$ and $C D_{i}$ denote the time difference and the cost difference threshold, respectively, of Route $j$ from Route $i$. Note that $T D_{i}$ and $C D_{i}$ can be treated as design parameters. Figure 3 shows the set of clustered routes.

Step 2. If the time-optimal route and the cost-optimal route are clustered, then stop the clustering procedure. Otherwise go back to Step $\mathbf{1}$ and recalculate incentive with the fewer number of route candidates.

\subsection{Determination of Incentive by Solving an Optimization Problem}

Once the departure point, the departure time, and the destination are designated by a user, the cloud system calculates $\left(T_{i}, C_{i}, E_{i}\right)$ of the possible routes. Suppose that there are $m$ possible routes from the departure point to the destination. For those routes, the likelihood $\psi_{i}$, $i=1, \ldots, m$, of choosing Route $i$ by the user can be determined from the data on the $T-C$ plane indicated by the color intensity as in Fig. 1. Our main idea is to shift the point $\left(T_{i}, C_{i}\right)$ by giving (adverse) incentive and changing the cost for Route $i$ so that energy efficient routes are more likely to be chosen by the user. Figure 4 shows the typical tendency of routes shifted by the incentive depending on the energy consumption of each route.

In order to derive an appropriate incentive for each route, we reformulate the minimization of energy consumption of each individual user as the minimization of average energy consumption. Specifically, we solve the optimization problem (1) or, equivalently, 


$$
\min _{\tilde{\psi}} \sum_{i=1}^{m} E_{i} \tilde{\psi}_{i}
$$

subject to

$$
\begin{aligned}
& \sum_{i=1}^{m} \psi_{i}=1, \quad \sum_{i=1}^{m} \tilde{\psi}_{i}=1 \\
& \tilde{\psi}_{i} \geq 0, \quad \tilde{C}_{i} \geq \epsilon_{i}, \quad \sum_{i=1}^{m} \Delta C_{i} \tilde{\psi}_{i} \leq \kappa,
\end{aligned}
$$

for $i=1, \ldots, m$, where $m$ is the number of candidate routes, $\tilde{\psi}_{i} \triangleq \psi_{i}+\alpha \Delta C_{i}, \tilde{C}_{i}\left(\triangleq C_{i}-\Delta C_{i}\right)$ is the cost including incentive, $\psi_{i}$ is the likelihood of choice by the users, $\epsilon_{i}$ is the minimum cost that the user has to pay when he/she takes Route $i, \alpha$ is the sensitivity for $\psi_{i}$ associated with the incentive $\Delta C_{i}$, and $\kappa$ denotes the net incentive funded by an organization such as the government or the institution running this navigation system. The role of this organization is to collect the funds from the society and to redistribute it in such a way that the society receives the benefit of the reduction of the amount of energy consumption.

Note that the objective function $\sum_{i=1}^{m} E_{i} \tilde{\psi}_{i}$ in (4) is equivalent to the expectation of energy consumption after assigning the incentive. As such, the optimization problem reduces to finding the optimal incentive to each of the candidate routes. In particular, the optimization problem (4)-(6) is equivalently reformulated as

$$
\min _{\left\{\Delta C_{i}\right\}} \sum_{i=1}^{m} E_{i}\left(\psi_{i}+\alpha \Delta C_{i}\right),
$$

subject to

$$
\begin{gathered}
\sum_{i=1}^{m} \Delta C_{i}=0, \\
-\frac{\psi_{i}}{\alpha} \leq \Delta C_{i} \leq \gamma C_{i}, \\
\sum_{i=1}^{m}\left(\psi_{i}+\alpha \Delta C_{i}\right) \Delta C_{i} \leq \kappa,
\end{gathered}
$$

along with (5), where $\gamma>0$ is such that $\gamma C_{i}=C_{i}-$ $\epsilon_{i}$. Since $E_{i}, \psi_{i}, i=1, \ldots, m$, are constants, it is now obvious that the optimization problem is the problem of finding the optimal incentive. Finally, it is important to note that this optimization problem reduces to a nonlinear programming problem such that some techniques in semidefinite programming (SDP) is extremely useful.

\subsection{Recalculation of Route Candidates}

As the final step, it is necessary to eliminate routes which are both time-consuming and costly relative to another route. Specifically, if $\operatorname{Route}_{i}\left(T_{i}, C_{i}, E_{i}\right)$ and $\operatorname{Route}_{j}\left(T_{j}, C_{j}, E_{j}\right.$ have the relationship

$$
T_{i}<T_{j}, \quad C_{i}<C_{j}
$$

then $\operatorname{Route}_{j}\left(T_{j}, C_{j}, E_{j}\right)$ has no advantage to leave as a candidate. On the $T-C$ plane, these meaningless routes appear upper right of another route candidate as shown in Fig. 5.

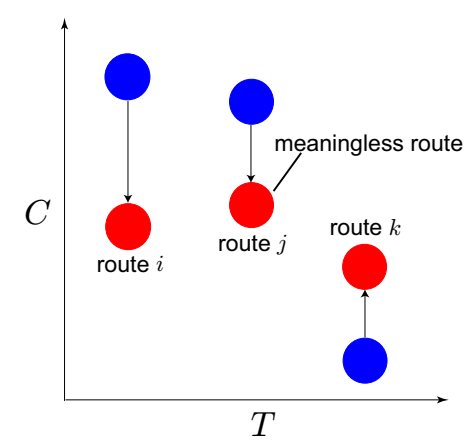

Fig. 5. Meaningless path. Route $j$ in red appears upper right of Route $i$ in red.

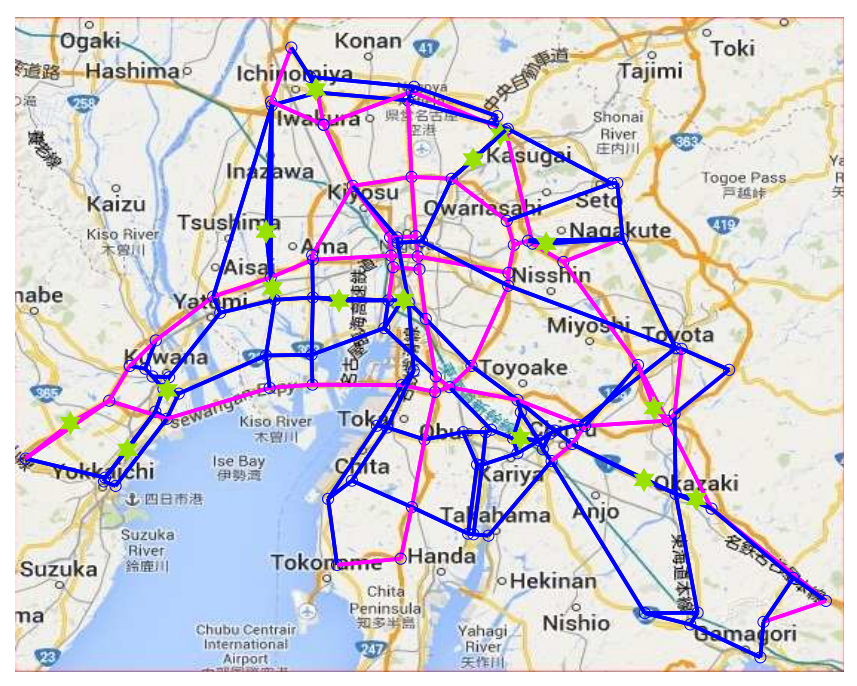

Fig. 6. Road map of Chukyo area in Japan. Magenta lines indicate tolled highway and blue lines indicate regular roads. Green marks indicate the charge points.

Table 1. Parameter settings

\begin{tabular}{|l||l|}
\hline Battery capacity & $24[\mathrm{kWh}]$ \\
\hline $\begin{array}{l}\text { Initial residual } \\
\text { battery amount }\end{array}$ & $24[\mathrm{kWh}]$ \\
\hline $\begin{array}{l}\text { Desirable residual } \\
\text { battery amount }\end{array}$ & $16[\mathrm{kWh}]$ \\
\hline Charging speed & $0.64[\mathrm{kWh} / \mathrm{min}]$ \\
\hline Charging price & 100 yen per $30 \mathrm{~min}$ \\
\hline Highway tolls & $\begin{array}{l}(150+\text { distance }[\mathrm{km}] \times 24.6) \\
\times 1.05[\text { yen }]\end{array}$ \\
\hline
\end{tabular}

\section{NUMERICAL SIMULATION}

In this section, we provide a numerical simulation where we apply the proposed framework for Chukyo (greater Nagoya) area in Japan. Specifically, we extract major roads (including tolled roads) and intersections in the area mapped as a graph of 130 nodes and 223 edges (Fig. 6).

For the simulation, we use the physical parameters listed )in Table 1 and the design parameters $\alpha=1.0 \times 10^{-4}$, $\gamma=0.8$, and $\kappa=30$. Furthermore, we set Kami-Ouchi as the departure point and Fuji-3chome as the destination.

We assume that the likelihood distribution of users representing the route selection tendency is given as follows. Let $T_{\text {min }}$ denote the minimum travel time and $C_{\text {min }}$ denote the minimum cost, which are obtained by the Dijkstra algo- 


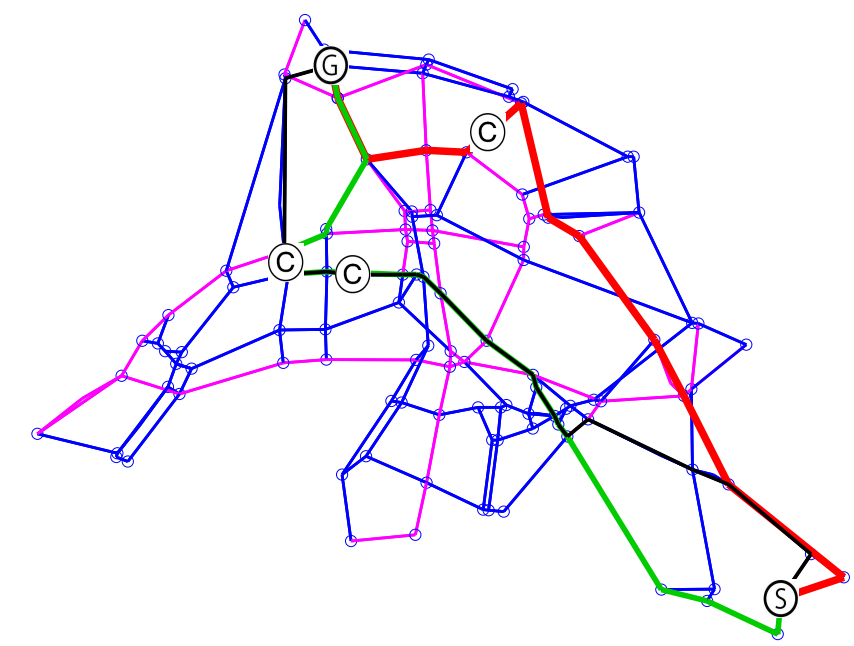

Fig. 7. Simulation result visualized on the graph shown in Fig. 6. The nodes $\mathrm{S}$ and $\mathrm{G}$ denote the start node and the goal (destination) node, respectively, and $\mathrm{C}$ indicates the charge points that the vehicle is to stop by on its way. Black route is the top-priority candidate. Green and red routes are the second and the third ones, respectively.

rithm. Now we consider the following curve as a reference line given by

$$
\left(T-\xi T_{\min }\right)\left(C-\eta C_{\min }\right)=\sigma
$$

where $\xi=0.8, \eta=0.8, \sigma=T_{\min } C_{\min }$, and assume that every route on this reference line is selected with the same probability by the users. Then it is supposed that the probability to select the Route $i$ is given as

$$
\psi_{i}=\frac{\frac{C_{i}}{C_{i}^{\prime}}}{\sum_{j=1}^{m} \frac{C_{j}}{C_{j}^{\prime}}},
$$

where $C_{i}^{\prime}$ denotes the cost obtained by projecting the traveling time and the cost of the route $i$ onto the reference line along the cost (vertical) axis. (It is important to note that this characterization is contrived for representing the color distribution of the $C-T$ diagram (Fig. 1) for the incentive calculation. The actual distribution of individual preferences should be obtained via questionnaire investigation and/or collecting data through real operation.)

The obtained results based on the above setting are shown in Figs. 7-10 and Tables 2 and 3. Figure 7 shows the three candidate routes obtained by the extended Dijkstra algorithm, where the black line denotes the top-priority candidate, the green line the second-priority candidate, and the red line the third-priority candidate. In this example, these solutions are obtained after 3 repetitions of Step 1 and Step 2 in Section 3.3. Figs. 8-10 show the results of three rounds of recalculation of route candidates. Specifically, in Figs. 8 and 9, which correspond to the first and the second rounds, respectively, we see that there exist meaningless routes as candidates in the sense that they need both higher cost and longer travel time compared with either of the other routes. On the other hand, in the third round shown in Fig. 10, such meaningless routes are eliminated. Figure 10 also shows that the cost of the route having larger energy consumption is relatively increased. As a result, as shown in Table 2, the probability

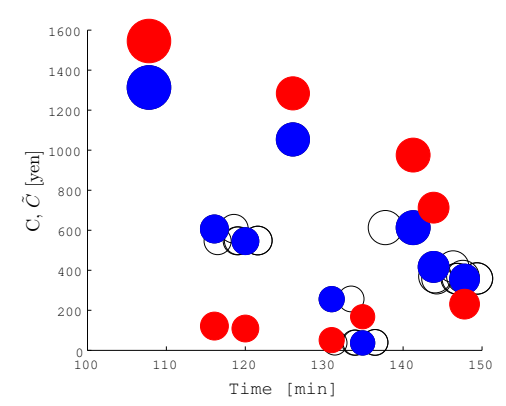

Fig. 8. $C$-T plane (after 1st optimization)

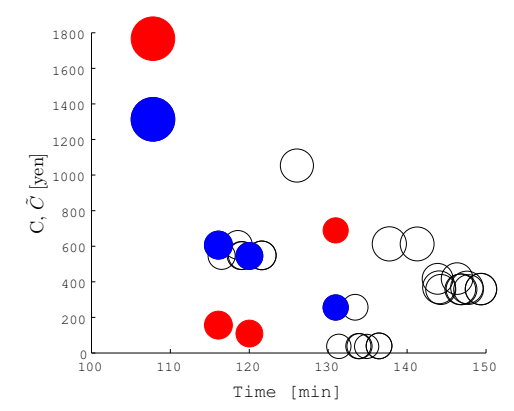

Fig. 9. $C-T$ plane (after 2nd optimization)

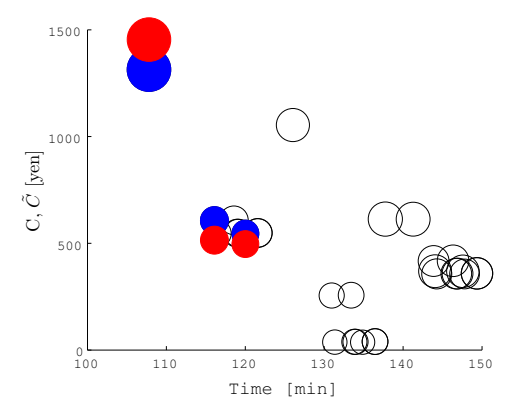

Fig. 10. $C-T$ plane (after 3rd optimization)

Table 2. Change of likelihood that the users take possible routes

\begin{tabular}{|c|c|c|c|}
\hline & 1st route & 2nd route & 3rd route \\
\hline \hline Without incentive & 0.4107 & 0.3860 & 0.2033 \\
\hline With incentive & 0.4156 & 0.3952 & 0.1892 \\
\hline
\end{tabular}

to select the top-priority candidate and the second-priority candidate is increased, while the probability to choose the third-priority candidate is decreased, by appropriately adding incentive to the expense that it costs for each route.

Next, we compare the above results with the case of the route associated with the minimum traveling time, which is usually suggested as one of the candidates in the current navigation system. Table 3 shows that the expectation of energy consumption for the proposed approach reduces $22 \%$ in comparison with the case of the route with the minimum travel time by sacrificing the travel time which increased by $7.20 \%$.

Third, we discuss the effectiveness of the proposed approach in a statistical way. We randomly select 110 pairs of the departure point and the destination whose distance in a straight line is more than $65[\mathrm{~km}]$. The average values of 110 results obtained under the same situation as the 
Table 3. Expected values of energy consumption, total travel time, and cost for the minimum-time route and the route with incentive

\begin{tabular}{|c|c|c|}
\hline Minimum-time route & With incentive & \\
\hline \hline \multicolumn{2}{|c|}{ Energy consumption [kWh] } & Reduction rate \\
\hline 28.24 & 22.24 & $21.53 \%$ \\
\hline \hline \multicolumn{2}{|c|}{ Travel time [min] } & Increasing rate \\
\hline 107.79 & 116.15 & $7.20 \%$ \\
\hline \hline \multicolumn{2}{|c|}{ Cost [yen] } & Reduction rate \\
\hline 1313.46 & 744.91 & $56.71 \%$ \\
\hline
\end{tabular}

Table 4. Aggregate expected values of energy consumption, total travel time, and cost for the minimum-time route and the route with incentive

\begin{tabular}{|c|c|c|}
\hline Minimum-time route & With incentive & \\
\hline \hline \multicolumn{2}{|c|}{ Energy consumption [kWh] } & Reduction rate \\
\hline 25.40 & 19.65 & $22.66 \%$ \\
\hline \hline \multicolumn{2}{|c|}{ Travel time [min] } & Increasing rate \\
\hline \hline 116.36 & 141.89 & $18.00 \%$ \\
\hline Cost [yen] & Reduction rate \\
\hline
\end{tabular}

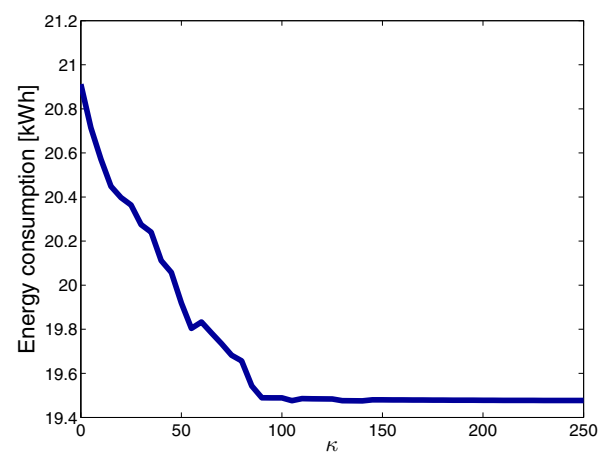

Fig. 11. Energy consumption versus $\kappa$

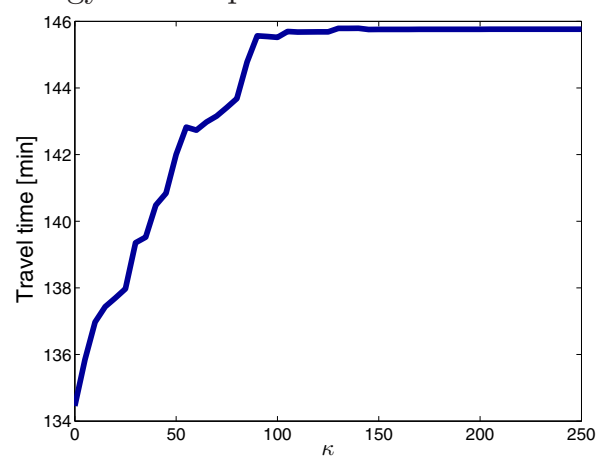

Fig. 12. Travel time versus $\kappa$

previous one as in Table 1 are shown in Table 4 . It turns out that the energy consumption reduces about $22 \%$ in this case compared to the case of the minimum-time route.

Finally, Figs. 11 and 12 show the relation between $\kappa$ and energy consumption and between $\kappa$ and travel time, respectively. Recall that $\kappa$ is the net incentive funded by the operating organization of this incentive based navigation system. As shown in Fig. 11 and 12, total energy consumption reduces and total travel time increases as $\kappa$ increases. This result implies that there is a trade- off relationship between the total energy consumption and the total travel time by changing the total fund supplied into the framework. In other words, if we increase the amount of fund $\kappa$ available in the framework so that more discount is possible for the users, the users are more likely to choose more energy efficient routes even though travel time increases.

\section{CONCLUSION}

This paper proposed an incentive based multi-objective optimization method for the use of EV navigation systems including battery charging, which changes tendency of users in selecting a route by an incentive approach to achieve suitable trade-off between global optimization and local optimization, namely, the entire energy consumption of EVs of all users and the QoL on travel time and cost of individual users simultaneously. Since the problem to be studied here involves uncertainty of human behavior, we assume that each user has a kind of probabilistic distribution in selecting a route. As pointed out in Section 4, one of the next important issues is to construct the $C$ $T$ diagram describing the tendency of users' preferences in terms of travel time and cost. Furthermore, the framework we proposed in this paper can be made useful by modifying how to treat the likelihood distribution that the users have in an appropriate way.

\section{REFERENCES}

A. Artmeier, J. Haselmayr, M. Leucker, and M. Sachenbacher. The shortest path problem revisited: optimal routing for electric vehicles. R. Dillmann et al. (Eds.): KI 2010: Advances in Artificial Intelligence, LNAI 6359, Springer, pp. 309-316 (2010)

E. Dijkstra. A note on two problems in connexion with graphs. Numerischce Mathematik. vol.1, pp.269-271, 1959.

J. Eisner, S. Funke, and S. Storandt. Optimal route planning for electric vehicles in large networks. Proc. of the 25th AAAI Conference on Artificial Intelligence (2011).

GoGoEV. http://ev.gogo.gs/, in Japanese.

T. Kanazawa, Y. Fukumoto, T. Ushio, and T. Misaka. Replicator dynamics with Pigovian subsidy and capitation tax. Nonlinear Analysis. vol.71, pp.e818-e826 (2009).

T. Kanazawa, T. Misaka, and T. Ushio. A control method of dynamic selfish routing based on a state-dependent tax. IEICE Trans. Fundamentals vol.E96, pp.1794-1802 (2013).

K. Kato. Note on optimal path for an electric vehicle. IEICE technical report, Theoretical Foundations of Computing. vol.109, pp.13-17 (2010).

Ministry of the Environment of Japan. A strategy for the spread of next-generation vehicles. http://www.env.go.jp/air/report/h2101/index.html, (2010), in Japanese.

U. F. Siddiqi, Y. Shiraishi, and S. M. Sait. Multi constrained route optimization for electric vehicles using SimE. Proc. of International Conference of Soft Computing and Pattern Recognition (SoCPaR), pp.376-383 (2011).

S. Storandt. Quick and energy-efficient routes: computing constrained shortest paths for electric vehicles Proc. of the 5th ACM SIGSPATIAL International Workshop on Computational Transportation Science, pp.20-25 (2012). 